\title{
Pressure and temperature dependence of the Raman phonons in isotopic $\gamma$-CuI
}

\author{
J. Serrano, ${ }^{1}$ M. Cardona, ${ }^{1}$ T. M. Ritter, ${ }^{2}$ B. A. Weinstein, ${ }^{3}$ A. Rubio, ${ }^{4}$ and C. T. Lin ${ }^{1}$ \\ ${ }^{1}$ Max-Planck-Institut für Festkörperforschung, Heisenbergstr. 1, D-70569 Stuttgart, Germany \\ ${ }^{2}$ UNC Pembroke, Dept. of Chemistry and Physics, Pembroke, North Carolina 28372 \\ ${ }^{3}$ SUNY Buffalo, Dept. of Physics, Buffalo, New York 14260 \\ ${ }^{4}$ Dpto. Física de Materiales, Facultad de Ciencias Químicas, Universidad del País Vasco, Centro Mixto CSIC-UPV/EHU \\ and Donostia International Physics Center, E-20018 San Sebastián, Basque Country, Spain
}

(Received 5 August 2002; published 5 December 2002)

\begin{abstract}
The transverse-optic (TO) phonons of zinc-blende-type $\mathrm{CuCl}(\gamma-\mathrm{CuCl})$ and the longitudinal-optic (LO) phonons of $\gamma-\mathrm{CuBr}$ exhibit striking anharmonic self-energy anomalies at the center of the Brillouin zone. We investigate here by Raman spectroscopy the dependence of the LO and TO phonons of $\gamma$-CuI on pressure, temperature, and isotopic mass of copper, in the search for related effects. We find that the pressure dependence of the TO phonon linewidth is qualitatively different at low temperature and close to room temperature. A model is developed to interpret these differences based on anharmonic decay into two and three phonons (two different channels). In order to make semiquantitative predictions we used the experimental pressure dependence of the TO phonons and that of phonons at the edge of the Brillouin zone that we obtained by linearresponse $a b$ initio local-density approximation calculations. We have also calculated the pressure dependence of the zone-edge phonons of $\gamma-\mathrm{CuCl}$ and $\gamma-\mathrm{CuBr}$ and compared it with the few existing experimental results. In this process, interesting anomalies concerning the transverse-acoustic phonons at the zone edge have been found.
\end{abstract}

DOI: $10.1103 /$ PhysRevB.66.245202

PACS number(s): 63.20.Ry, 63.20.Kr, 62.50.+p, 78.30.Hv

\section{INTRODUCTION}

Krauzman et al. ${ }^{1}$ discussed the anomalous lineshape of the transverse-optic (TO) Raman phonons of $\mathrm{CuCl}$ in the zinc-blende crystal structure $(\gamma-\mathrm{CuCl})$ and attributed it to strong, Fermi-resonance-like, anharmonic coupling with the corresponding two-phonon acoustic density of states (DOS). This assignment has been contested by several authors. ${ }^{2-4}$ However, recent work with isotopically substituted samples ${ }^{5}$ and as a function of pressure ${ }^{6}$ has rather conclusively confirmed the Fermi resonance model. No such anomaly is believed to exist for the TO phonons of the isostructural $\gamma$-CuBr. ${ }^{7}$ Recently, however, a similar anomaly has been found for the corresponding longitudinal-optic (LO) Raman phonon of $\gamma-\mathrm{CuBr}^{8}{ }^{8}$ The shift of the anomaly from the TO phonons in $\gamma-\mathrm{CuCl}$ to the $\mathrm{LO}$ phonons in $\gamma-\mathrm{CuBr}$ can be qualitatively understood with a rather simple argument: The $\mathrm{TO}$ and $\mathrm{LO}$ phonons of $\gamma-\mathrm{CuCl}$ shift down in frequency when chlorine is replaced by bromine, because of the strong increase in reduced mass. However, the acoustic modes which give rise to the two-phonon DOS required for the Fermi anomaly are hardly shifted by this substitution since their reduced mass is mainly that of copper. This leads to the disappearance of the $\mathrm{TO}_{\Gamma^{-}}(\mathrm{TA}+\mathrm{LA})$ degeneracy of $\gamma-\mathrm{CuCl}$ which is replaced in $\mathrm{CuBr}$ by a $\mathrm{LO}_{\Gamma^{-}}(\mathrm{TA}+\mathrm{LA})$ degeneracy. Similar arguments lead to the lifting of the Fermi resonances of both TO and LO Raman phonons in CuI, a feature which is already evident in the theoretical work of Kanellis et al. ${ }^{7}$

The fact that the Fermi resonance of Raman phonons present in $\gamma-\mathrm{CuCl}$ and in $\gamma-\mathrm{CuBr}$ is strongly affected by pressure and by isotopic mass substitution has led us, in the present work, to investigate the dependence on those parameters of the TO and LO phonons of $\gamma$-CuI at the center of the Brillouin zone ( $\Gamma$ point) by Raman spectroscopy. As an ad- ditional parameter which may reveal possible Raman resonances, we have varied the temperature between 10 and 300 $\mathrm{K}$ (at higher temperatures the strongly broadened TO and LO peaks are difficult to measure). At low pressure, we have seen a strong increase in the linewidth of both phonons, especially that of the TO ones which broaden from $\approx 0.5 \mathrm{~cm}^{-1}$ at $10 \mathrm{~K}$ to $12 \mathrm{~cm}^{-1}$ at $300 \mathrm{~K}$. The understanding of this strongly nonlinear temperature dependence requires the presence of two anharmonic decay channels, one involving decay into two phonons, the other into three. The corresponding temperature dependence of the LO Raman phonons can, however, be understood on the basis of mainly anharmonic decay into two phonons.

We have also investigated the anharmonic temperature shift of the LO and TO Raman phonons. The shifts of the TO and LO phonons correspond, as usual, to third- and fourthorder anharmonic vertices of the self-energy which cannot be separated on an experimental basis.

Some anomalies in the phonon line shape arise with temperature for both $\mathrm{TO}$ and $\mathrm{LO}$ modes and are interpreted here with the aid of self-energies calculated from the one- and two-phonon (sum as well as difference) DOS. A rigid-ion force constants model ${ }^{10}$ (RIM) was used to obtain the DOS from the phonon dispersions reported by inelastic neutron scattering experiments. ${ }^{11}$ The application of these densities of states to the understanding of the observed line shapes and line shifts at different pressure and temperature conditions requires the knowledge of the mode Grüneisen parameters of the TO and LO phonons at $\Gamma$ (which follow from our pressure experiments) and those of the corresponding two- and three-phonon critical points involved in the anharmonic processes. About the latter Grüneisen parameters, little experimental information is available. We have therefore under- 
taken an $a b$ initio local-density approximation (LDA) calculation of the phonon frequencies and their variation with volume for the three cuprous halides. The phonon frequencies so obtained are typically $10 \%$ lower than the known experimental results. The corresponding Grüneisen parameters agree semiquantitatively with the few available experimental data. In particular, they reproduce the negative values observed for the zone-edge transverse-acoustic modes of $\gamma-\mathrm{CuCl}$ and $\gamma-\mathrm{CuBr}$, and also the strong decrease in the absolute value for $\gamma$-CuI, for which it is very small and possibly positive, a trend opposite to that observed for some II-VI compounds.

\section{EXPERIMENTAL DETAILS}

The experiments were performed on $\mathrm{CuI}$ samples grown from the vapor phase using a procedure described elsewhere. ${ }^{12-14}$ Crystals were grown using natural copper $\left(69 \%{ }^{63} \mathrm{Cu}, 31 \%{ }^{65} \mathrm{Cu}\right)$ as well as isotopically enriched (to better than $99 \%){ }^{63} \mathrm{Cu}$ and ${ }^{65} \mathrm{Cu}$. Iodine, unfortunately, has only one stable isotope $\left({ }^{127} \mathrm{I}\right)$. The crystals we used were basically the same as employed in Ref. 13 to study the dependence of the band gap on temperature and isotopic mass.

\section{A. Temperature dependence of Raman phonons}

Measurements of the Raman phonons in CuI vs temperature were performed using two different setups. First we employed a Janis cryostat to ensure that the temperature would be lower than $2 \mathrm{~K}$ by inmersing the samples in liquid $\mathrm{He}$. However, for the measurement of the temperature dependence a microscope cryostat equipped with a cold finger was used. The $6470.9 \AA$ line of a mixed $\mathrm{Ar}^{+}-\mathrm{Kr}^{+}$gas laser was utilized as excitation source. The backscattered light was analyzed by a XY-Dilor triple grating spectrometer operating in additive mode for the low- $T$ measurements. It was detected by a charge-coupled device (CCD) cooled down with liquid nitrogen. In the additive mode this CCD allows a spectral resolution of $\approx 0.4 \mathrm{~cm}^{-1}$ [full width at half maximum (FWHM)]. Since the Raman spectra broaden enormously with temperature and the additive mode of the spectrometer in conjunction with the CCD detector covers a spectral range of only $\approx 200 \mathrm{~cm}^{-1}$, we operated the spectrometer in the subtractive mode for investigating the temperature dependence in the full range between 6 and $300 \mathrm{~K}$; in this case the resolution was limited to $\approx 0.7 \mathrm{~cm}^{-1}$. Due to the small thickness of the ${ }^{65} \mathrm{CuI}$ sample, the opening of the slits of the monochromator had to be increased, yielding a slightly lower resolution of $\approx 1.1 \mathrm{~cm}^{-1}$.

\section{B. Pressure dependence of Raman phonons}

A diamond-anvil cell (DAC) operated with $\mathrm{He}$ as the pressure medium was employed to study the pressure dependence of the Raman phonons in $\mathrm{CuI}$ in the range between 0 and $2 \mathrm{GPa}$. Above that pressure $\gamma$-CuI undergoes a phase transition ${ }^{15}$ to a tetragonal phase that is not of interest here. We used the 6470.9- $\AA$ line of a $\mathrm{Kr}^{+}$-ion laser as excitation source and a double grating spectrometer to analyze the scattered light which was collected by a Princeton Instruments

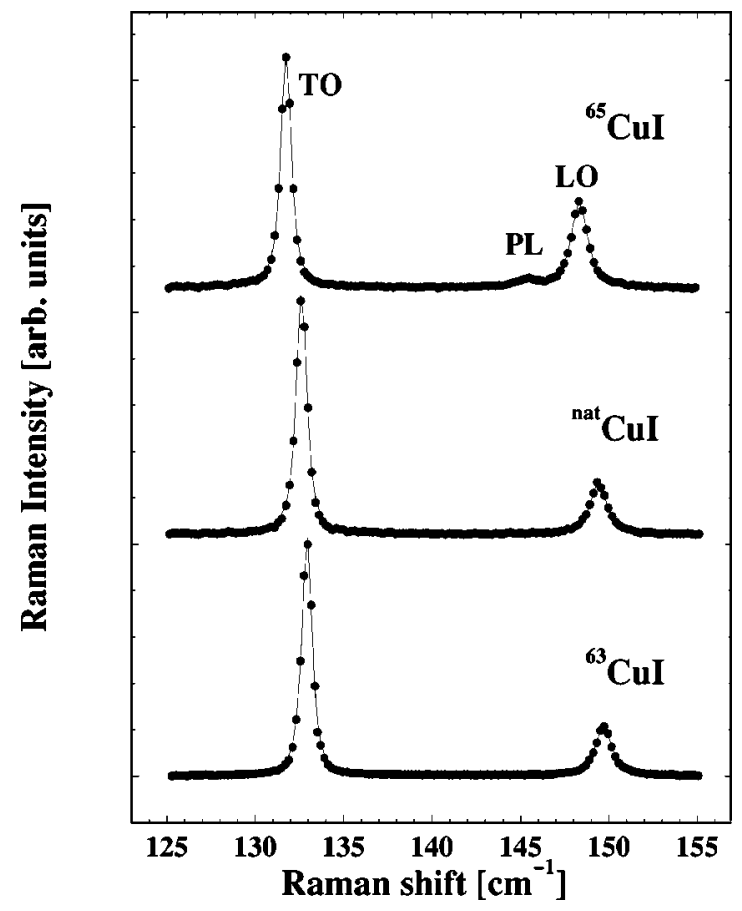

FIG. 1. Raman spectra of isotopically tailored and of natural $\mathrm{CuI}$ at $2 \mathrm{~K}$. The difference in intensity between the TO and the LO peaks is a clear indication of the (111) orientation of the samples (Ref. 21). A laser plasma line $(P L)$ can be seen at $\approx 145 \mathrm{~cm}^{-1}$.

cooled multichannel detector consisting of Peltier-cooled photocathode and microchannel plate intensifer, with a Sidiode array readout. With this setup a spectral resolution of $\approx 1 \mathrm{~cm}^{-1}$ (FWHM) was achieved. Several samples were used for these measurements, of typical sizes of $60 \times 60$ $\times 30 \mu \mathrm{m}$. They were loaded under a microscope in inconel gaskets together with a small ruby crystal utilized as reference for the determination of pressure. ${ }^{16,17}$ The pressure was applied by a hydraulic pump operated with oil which transferred the force to a steel tube pushing the upper diamond towards the lower one. The temperature was varyied by changing the flux of He exchange gas into the Janis cryostat and was controlled independently and measured to a precision of $\pm 1 \mathrm{~K}$ by two silicon sensors placed in thermal contact with the DAC near the position of the diamonds. More details of this cryogenic DAC system are given elsewhere. ${ }^{18}$

The spectra of both pressure and temperature dependent runs were analyzed by performing a fit with a Voigt function having a Gaussian contribution of FWHM equal to the spectral resolution. This was determined in all cases from the width of the 6532.9- $\AA$ emission line obtained from a lowpressure neon lamp used for calibration. Such fit procedure can be justified by the excellent resolution achieved in both pressure and temperature experiments. ${ }^{19}$

\section{RESULTS}

\section{A. Low-temperature dependence of Raman phonons on isotopic mass}

Figure 1 displays the Raman spectra of $\mathrm{CuI}$ at $2 \mathrm{~K}$ for the different isotopic compositions. Both phonon lines are rather 

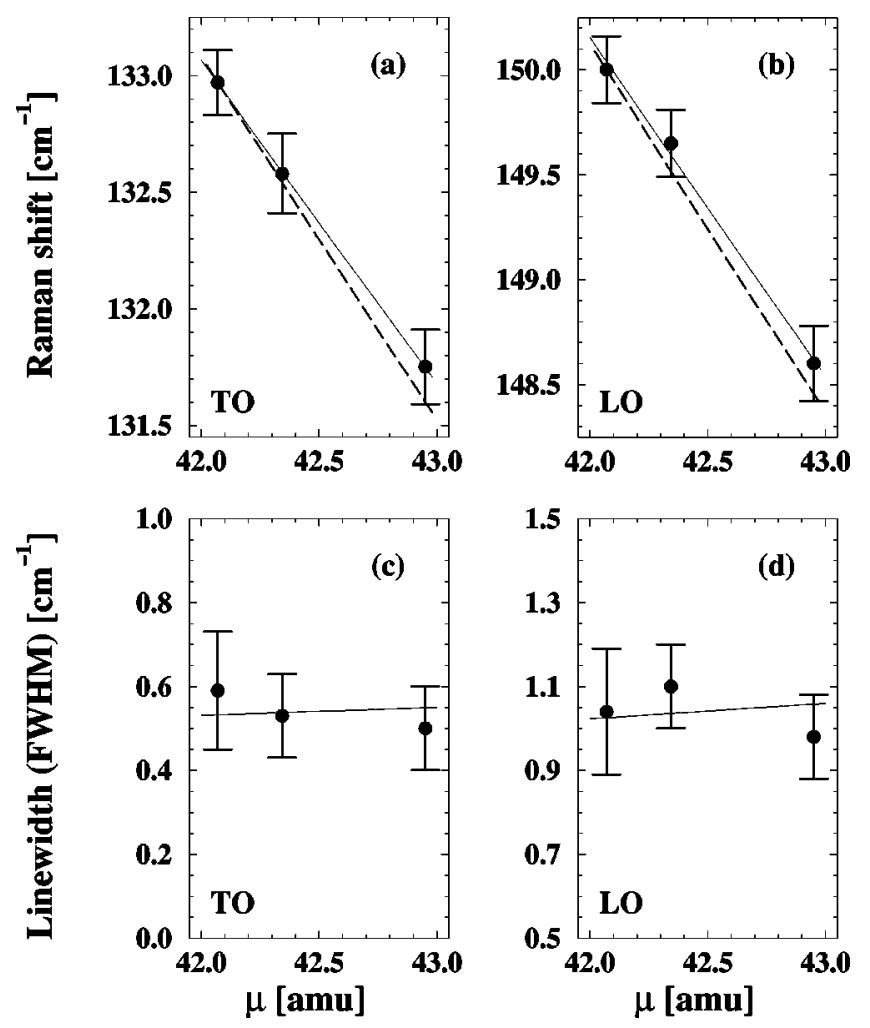

FIG. 2. Mass dependence of the phonon frequencies, (a) and (b), and linewidths (FWHM), (c) and (d), of the $2 \mathrm{~K}$ spectra of Fig. 1. The dashed lines represent the $\approx \mu^{-1 / 2}$ behavior expected for the harmonic virtual crystal approximation (VCA) using ${ }^{63} \mathrm{CuI}$ as reference. The solid lines display fits with the mass dependence predicted by Eqs. (1) and (2) for the phonon frequencies and linewidths, respectively.

sharp, although the longitudinal phonon peak appears to be broader and weaker. The weak structure, denoted by $P L$, observed at $\approx 145 \mathrm{~cm}^{-1}$ in the spectrum of ${ }^{65} \mathrm{CuI}$ reflects a plasma line produced by the laser which could not be avoided with a filter due to the small thickness of this sample and the correspondingly low Raman signal. A shift of the phonon peaks to lower frequencies appears when the copper mass increases. The zone center modes behave like $\approx \mu^{-1 / 2}$ with $\mu$ the reduced mass obtained from the average masses of both constituents, thus in agreement with the harmonic virtual crystal approximation (VCA).

A more detailed analysis of these spectra is performed in Fig. 2, where the Raman shifts and phonon linewidths are shown for both TO and LO phonons for copper-isotopeenriched and natural $\mathrm{CuI}$. The dashed lines represent the $\mu^{-1 / 2}$ behavior predicted by the harmonic VCA, where we have taken the ${ }^{63} \mathrm{CuI}$ sample as reference. We can observe that, in spite of the good agreement, the Raman frequency shift with increasing copper mass is marginally lower than the prediction of the harmonic theory.

In order to take into account anharmonicity to lowest order, the dependences of the Raman frequencies and linewidths of monatomic crystals on isotopic mass $M$ have been described in the past with the expressions $\omega=A M^{-1 / 2}$ $-B M^{-1}$ (e.g., Si, Ref. 20) and $\Gamma(\omega)=C M^{-1}$. These equa- tions can be extended to diatomic, zinc-blende-type crystals like $\gamma$-CuI by assuming that all acoustic phonons into which the Raman mode decays depend solely on the mass of the heavier atom. Under this hypothesis the linewidth of the Raman phonons of $\mathrm{CuI}$ would be given at low temperature by ${ }^{19}$

$$
\begin{aligned}
\Gamma(\omega) & =C\left|u_{I}^{\Gamma} u_{I}^{a c} u_{I}^{a c}\right|^{2} \times \rho^{(2)}(\omega) \\
& =C \mu^{3 / 2} M_{I}^{-5 / 2},
\end{aligned}
$$

where $u_{I}^{\Gamma}$ and $u_{I}^{a c}$ denote the iodine component of the atomic displacements for the Raman phonon and the acoustic phonons into which the former decays, respectively, and $\rho^{(2)}(\omega)$ is the corresponding two-phonon DOS, assumed to vary with the mass as $\approx M_{I}^{1 / 2}$. We obtained from the fit with Eq. (1) to the TO (LO) phonon frequencies $B$ $=353.8$ (681.6) $\mathrm{cm}^{-1}$ amu. Note that Eq. (1) leads to the result that the linewidth increases with increasing $\mu$, i.e., with increasing copper mass $M_{C u}$, contrary to the decrease with mass found for monatomic crystals. This seemingly paradoxical prediction can be understood when one considers that the anharmonic matrix element is determined by the component of the displacement of the Raman phonon on the iodine atoms. This displacement increases with increasing $M_{C u}$.

The anharmonic contribution to the dependence of the phonon frequencies on the copper mass is more complicated to estimate, since it corresponds to an integration involving $\rho^{(2)}(\omega)$ over the entire frequency space. If we admit as a rough simplification that most of the two-phonon DOS is concentrated at $\approx 2 \omega_{T O}$ and has mainly copperlike character, the following expression including the lowest term in perturbation theory for the anharmonic contribution can be derived:

$$
\omega=A \mu^{-1 / 2}-B \frac{\mu^{3 / 2} M_{C u}^{-3}}{2 M_{C u}^{-1 / 2}-\mu^{-1 / 2}} .
$$

We obtained for the TO mode of $\mathrm{CuI}$ at $2 \mathrm{~K} A$ $=898.1 \mathrm{~cm}^{-1} \mathrm{amu}^{1 / 2}$ and $B=491.8 \mathrm{~cm}^{-1} \mathrm{amu}$, and for the LO mode $A=1002.5 \mathrm{~cm}^{-1} \mathrm{amu}^{1 / 2}$ and $B$ $=404.6 \mathrm{~cm}^{-1}$ amu. Despite the large error bars, we can extract from these values of $B$ using Eq. (2) a zero-temperature anharmonic renormalization $\Delta_{1}=-5 \pm 3 \mathrm{~cm}^{-1} \quad(-4$ $\pm 3 \mathrm{~cm}^{-1}$ ) for the TO (LO) phonons. These values agree within the error bars with those obtained from measurements of the phonon frequencies vs temperature: $-2.7 \mathrm{~cm}^{-1}$ for the TO mode and $-2.3 \mathrm{~cm}^{-1}$ for the LO phonon. This oversimplified model can be extended by using for $\rho^{(2)}(\omega)$ an additional oscillator with iodinelike character located at $\approx \frac{2}{3} \omega_{T O}$, which can lead to a slightly better agreement between the renormalization obtained by varying the isotopic mass and that extrapolating to $T=0 \mathrm{~K}$ the linear temperature dependence of the phonon frequencies found at high temperature. However, the error bars of the measurements do not warrant the use of this extended model.

\section{B. Temperature dependence of the anharmonic self-energies}

Because of the strong similarity of the spectra for the three isotopic compositions shown in Fig. 1, we only per- 


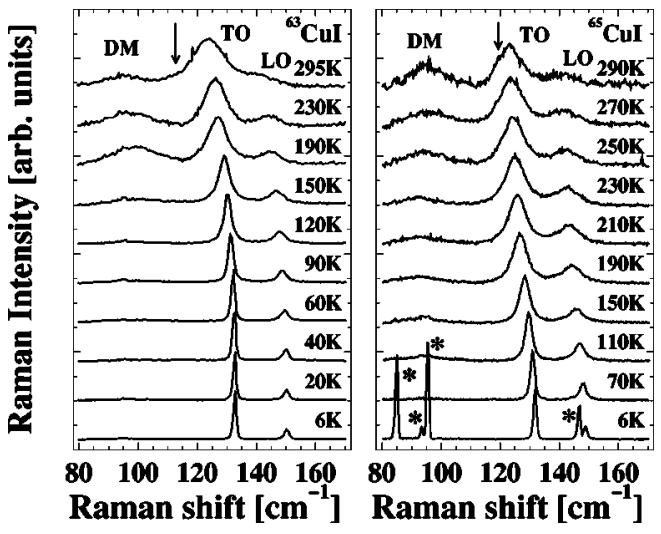

FIG. 3. Raman spectra of ${ }^{63} \mathrm{CuI}$ and ${ }^{65} \mathrm{CuI}$ shown for different temperatures. A broad feature connected with a van Hove singularity of the two-phonon difference DOS ( $D M$ stands for difference mode) appears for temperatures above $150 \mathrm{~K}$ at $\approx 95 \mathrm{~cm}^{-1}$. Note the dramatic broadening of both TO and LO peaks and the shoulder of the LO mode rising at low frequencies with increasing temperature. Peaks denoted by an asterisk in the ${ }^{65} \mathrm{CuI}$ spectra represent calibration lines from a neon lamp.

formed detailed temperature runs, between 6 and $300 \mathrm{~K}$, for ${ }^{63} \mathrm{CuI}$ and ${ }^{65} \mathrm{CuI}$. Typical data are shown in Fig. 3 at different temperatures. A broad structure is observed to rise with temperature at $\approx 95 \mathrm{~cm}^{-1}$ which can be related to a strong van Hove singularity in the two-phonon difference DOS; this will be discuss further in Sec. V. Moreover, both TO and LO modes experience a large increase of linewidth with temperature and a shoulder appears at the low-energy side of the LO phonon above $150 \mathrm{~K}$. This shoulder evolves with temperature in such a way that at room temperature TO and LO peaks strongly overlap for both isotopic compositions. Note that also the TO phonon displays an asymmetric line shape above $190 \mathrm{~K}$ for ${ }^{65} \mathrm{CuI}$, with more spectral weight at low frequencies, and a shoulder appears at $\approx 120 \mathrm{~cm}^{-1}$. For ${ }^{63} \mathrm{CuI}$ no measurements were performed between $230 \mathrm{~K}$ and room temperature, but the asymmetry can be noticed already at $150 \mathrm{~K}$. The fact that the integrated intensity of the TO peak is larger by a factor of 3 than that of the corresponding LO peak reflects the $\{111\}$ orientation of the measured surface. $^{21}$ This, added to the strong broadening at $300 \mathrm{~K}$, is the reason why the LO peak barely appears in many of the published spectra of $\gamma$-CuI which must have been taken on $\{111\}$ surfaces. $^{2,15,22,23}$ Figures 1 and 2 indicate that the TO peak is narrower $\left[\Gamma(\mathrm{FWHM}) \approx 0.5 \mathrm{~cm}^{-1}\right]$ at low temperatures than the corresponding LO peak [ $Г(\mathrm{FWHM})$ $\left.\approx 1 \mathrm{~cm}^{-1}\right]$, whereas at $300 \mathrm{~K}$ both widths are nearly equal for ${ }^{65} \mathrm{CuI}$. This fact will lead below to the necessity of introducing different decay channels for the interpretation of the measured temperature dependence of these linewidths, plotted in Fig. 4. This figure shows for ${ }^{63} \mathrm{CuI}$ a crossover in the temperature dependence of the TO and LO linewidths at about $170 \mathrm{~K}$. At $6 \mathrm{~K}$ the TO width (FWHM) is a factor of 2 smaller than the corresponding LO width, whereas it is about $30 \%$ larger at $300 \mathrm{~K}$, a fact which suggests different decay mechanisms for the two Raman phonons. For ${ }^{65} \mathrm{CuI}$ we do not observe such a crossover although the relative increase of the TO linewidth is similar to that obtained in ${ }^{63} \mathrm{CuI}$.

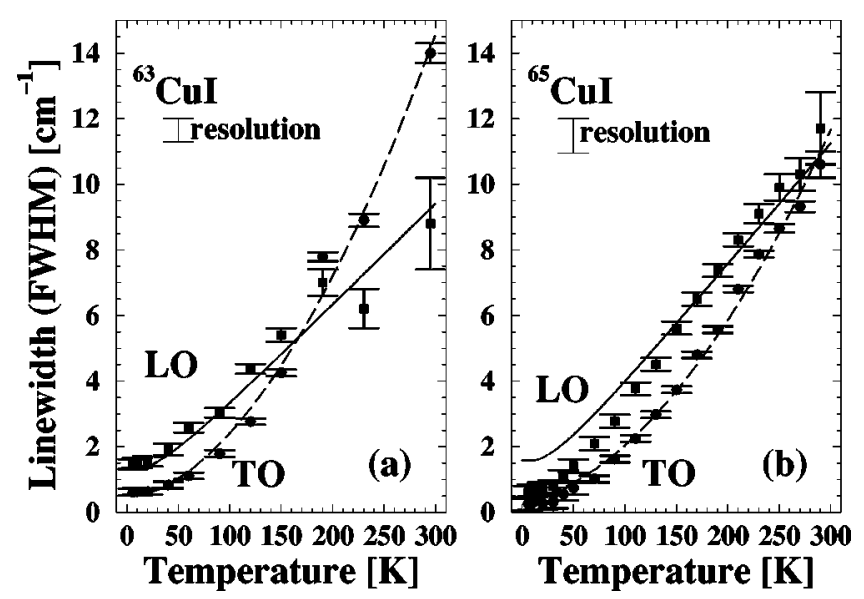

FIG. 4. Temperature dependence of the linewidth (FWHM) of TO and LO phonons corresponding to Fig. 3 after deconvoluting the spectrometer resolution using a Voigt profile. The solid lines show a fit of the LO peak with the assumption of a two-channel decay with frequencies given in Table I, whereas for the TO mode a term corresponding to decay into three phonons was added (dashed lines). The poor agreement with the fit for the LO phonon in ${ }^{65} \mathrm{CuI}$ can be partially attributed to the lower resolution used for this sample, which makes the fit with a Voigt profile rather inaccurate.

We have performed a fit of the LO linewidth with the expression

$$
\Gamma_{\mathrm{LO}}(T)=\Gamma_{1}\left[1+n_{B E}\left(\omega_{1}, T\right)+n_{B E}\left(\omega_{2}, T\right)\right],
$$

where $n_{B E}\left(\omega_{i}, T\right)$ is the corresponding Bose-Einstein factor with frequency $\omega_{i}$, and $\omega_{1}$ and $\omega_{2}$ are the frequencies of the phonons into which the LO phonon frequency $\omega_{\mathrm{LO}}$, decays, with the constraint $\omega_{\mathrm{LO}}=\omega_{1}+\omega_{2}$ imposed by energy conservation. The fit was performed for $\omega_{1}=110 \mathrm{~cm}^{-1}$ and $\omega_{2}$ $=40 \mathrm{~cm}^{-1}$, as suggested by the experimental dispersion relations. ${ }^{11}$ These values were taken to be averages of the experimental $^{24}$ frequencies for the TA and LA modes at the $X$ and $L$ points (see Table II). ${ }^{25}$ We avoid in this manner the so-called Klemens ansatz ${ }^{19,26}\left(\omega_{1}=\omega_{2}=\omega_{\mathrm{LO}} / 2\right)$ for which the density of two-phonon states would be very low (see Fig. 10.15b of Ref. 24 and Fig. 3 of Ref. 7).

The TO linewidth data of Fig. 4 show a very weak increase with temperature at low temperature, followed by a strong, nearly quadratic increase above $100 \mathrm{~K}$. This trend cannot be fitted with Eq. (3) which leads to an almost linear behavior down to rather low temperatures. We have therefore included in Eq. (3) a term representing the decay of the TO phonon into three phonons, which is quadratic vs temperature at high temperatures. This higher-order anharmonic contribution has also been used in Ref. 27 to explain the quadratic dependence on temperature of the LO phonons in cubic BN at high temperatures. We thus write

$$
\begin{aligned}
\Gamma_{\mathrm{TO}}(T)= & \Gamma_{1}\left[1+n_{B E}\left(\omega_{1}, T\right)+n_{B E}\left(\omega_{2}, T\right)\right] \\
& +\Gamma_{2}\left[1+3 n_{B E}\left(\omega_{3}, T\right)+3 n_{B E}^{2}\left(\omega_{3}, T\right)\right],
\end{aligned}
$$

where, in order to reduce the number of free parameters, we have taken $\omega_{1}=97 \mathrm{~cm}^{-1}$ and $\omega_{2}=35 \mathrm{~cm}^{-1}$, scaled from those used for the LO linewidth so as to fulfill energy con- 
TABLE I. Parameters obtained from the fit of the temperature dependence of the TO and LO phonon linewidths and frequencies of $\mathrm{CuI}$. The phonon frequencies were fitted using the two-phonon decay model with equal acoustic frequencies (Klemens ansatz) of Eq. (5). Phonon linewidths were reproduced by fits with Eqs. (3) and (4), where we used for the LO mode $\omega_{1}=40 \mathrm{~cm}^{-1}$ and $\omega_{2}$ $=110 \mathrm{~cm}^{-1}$, representing the maxima of transverse and longitudinal acoustic densities of states (Ref. 24), respectively, and the correspondingly scaled frequencies for the TO modes. $\Delta_{1}$ represent the zero-temperature anharmonic frequency renormalization obtained from Fig. 5. All parameters are given in $\mathrm{cm}^{-1}$.

\begin{tabular}{lcccccc}
\hline \hline & & $\omega_{0}$ & $\Delta_{1}$ & $\omega_{1}$ & $\Gamma_{1}$ & $\Gamma_{2}$ \\
\hline${ }^{63} \mathrm{CuI}$ & TO & 135.27 & 2.50 & 88.7 & 0.34 & 0.17 \\
& LO & 152.85 & 2.64 & 95.5 & 1.31 & \\
${ }^{65} \mathrm{CuI}$ & TO & 134.60 & 2.81 & 98.2 & 0.33 & 0.13 \\
& LO & 150.85 & 1.97 & 82.0 & 1.57 & \\
\hline \hline
\end{tabular}

servation, i.e., $\omega_{1}+\omega_{2}=\omega_{T O}$. These values correspond approximately to the frequencies of the TA and LA modes at the $L$ point of the Brillouin zone. We have also made the hypothesis $\omega_{3}=\omega_{\mathrm{TO}} / 3 \approx 44 \mathrm{~cm}^{-1}$, similar to that of Klemens for the decay into two phonons. This ansatz will also be justified in Sec. $\mathrm{V}$ as related to a strong critical point found in the one-phonon density of states at the frequency $\omega_{\mathrm{TO}} / 3$. The parameters of the fits of Fig. 4 are given in Table I.

At this point we should mention that Raman data of $\mathrm{Fu}-$ kumoto et al. ${ }^{23}$ show for the TO and LO phonons of $\gamma$-CuI at $300-\mathrm{K}$ linewidths of about $12 \mathrm{~cm}^{-1}$ (FWHM), which agree within error with those of Fig. 4. At $4 \mathrm{~K}$, however, their LO phonon spectra have a linewidth of $1.8 \mathrm{~cm}^{-1}$ and the corresponding width of the TO phonon spectra is $1.6 \mathrm{~cm}^{-1}$, in contrast with the results of our Figs. 2 and 4. We believe that this discrepancy arises from an instrumental width of about $1 \mathrm{~cm}^{-1}$ which was not corrected for (as stated by them).

The temperature shifts (corresponding to the real part of the anharmonic self-energy) obtained from fits to the spectra of Fig. 3 are shown in Fig. 5 for the TO and LO phonons. The experimental points have been fitted (solid lines) with an expression similar to Eq. (3), whereby in this case one

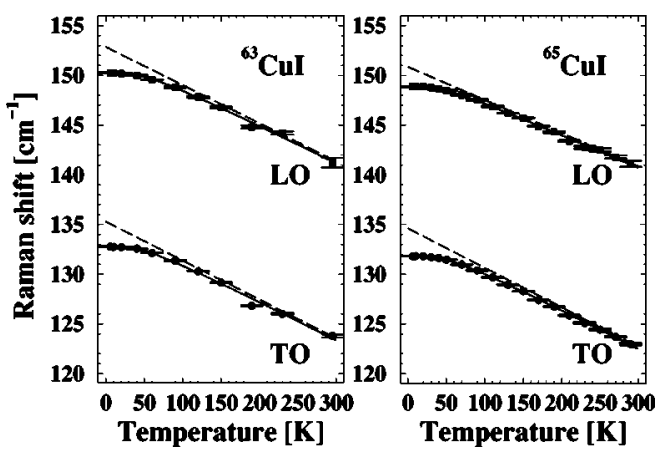

FIG. 5. Temperature dependence of the phonon frequencies of the zone-center modes in ${ }^{63} \mathrm{CuI}$ and ${ }^{65} \mathrm{CuI}$. The solid lines represent a fit with Eq. (5) (see text). The dashed lines display the extrapolation to $0 \mathrm{~K}$ of the linear dependence found at high temperatures. The parameters of the fit are listed in Table I.
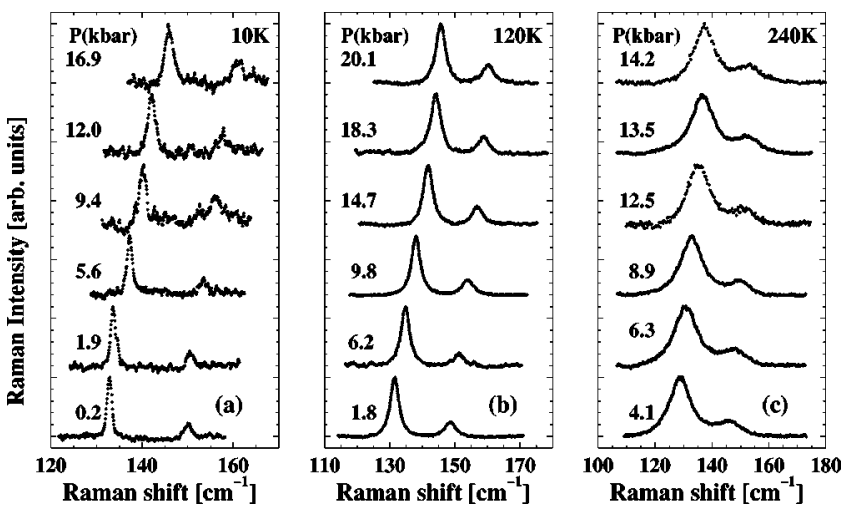

FIG. 6. Pressure dependence of the Raman spectra of ${ }^{63} \mathrm{CuI}$ at $10 \mathrm{~K}$ (a), $120 \mathrm{~K}$ (b), and $240 \mathrm{~K}$ (c). Note the broadening of the TO linewidth with increasing pressure observed at $10 \mathrm{~K}$.

should not fix the values of $\omega_{1}$ and $\omega_{2}$ by energy conservation since the intermediate states are energy nonconserving virtual transitions. This leaves us with two independent fit parameters, $\omega_{1}$ and $\omega_{2}$, representing average frequencies. Attempts to fit these parameters independently revealed that not enough experimental information is available (in view of the error bars in the experimental frequencies). We therefore make a Klemens-like ansatz $\omega_{1}=\omega_{2}$, whereby $\omega_{1}$ need not be $\omega_{\mathrm{TO}} / 2\left(\right.$ nor $\left.\omega_{\mathrm{LO}} / 2\right)$ for the fit of the TO (LO) phonons,

$$
\omega=\omega_{0}-\Delta_{1}\left[1+2 n_{B E}\left(\omega_{1}, T\right)\right] .
$$

An excellent fit is obtained for both TO and LO frequencies with $\omega_{1}=\omega_{2} \approx 90 \mathrm{~cm}^{-1}$; the specific values of $\Delta_{1}$ and $\omega_{1}$ obtained from the fit are displayed in Table I. These values agree qualitatively with the ad hoc assumption of a single oscillator at $2 \omega_{T O}$ made in Eq. (2) to explain the slight deviation of the reduced mass behavior expected from the VCA observed in Fig. 2 for low temperatures.

\section{Pressure dependence of the Raman phonons}

We have investigated the pressure dependence of the Raman phonons of ${ }^{63} \mathrm{CuI}$ at three different temperatures in order to buttress the interpretation of the changes in line shape and the line shift of the phonons and resonances observed in Fig. 3 at ambient pressure. The dependence of the Raman spectra of $\gamma$-CuI on pressure (up to $\approx 20 \mathrm{kbar}$ ) is shown in Figs. 6(a)-6(c), as measured at 10, 120, and $240 \mathrm{~K}$, respectively. The following trends are apparent:

(i) At $10 \mathrm{~K}$ both, the TO and LO linewidths increase with increasing pressure, the increase being difficult to measure quantitatively for the rather weak LO peak.

(ii) At $120 \mathrm{~K}$ it is not possible to detect a variation of the width of the weak LO peak with pressure within experimental scatter. The linewidth of the TO peak, however, increases by about $15 \%$ from 0.2 to $2.0 \mathrm{GPa}$. This increase is considerably less (a factor of three) than that found at $10 \mathrm{~K}$.

(iii) At $240 \mathrm{~K}$ the TO linewidth remains nearly independent of pressure at $9.5 \pm 0.5 \mathrm{~cm}^{-1}$. TO and LO peaks almost overlap like in the high-temperature spectra displayed in Fig. 

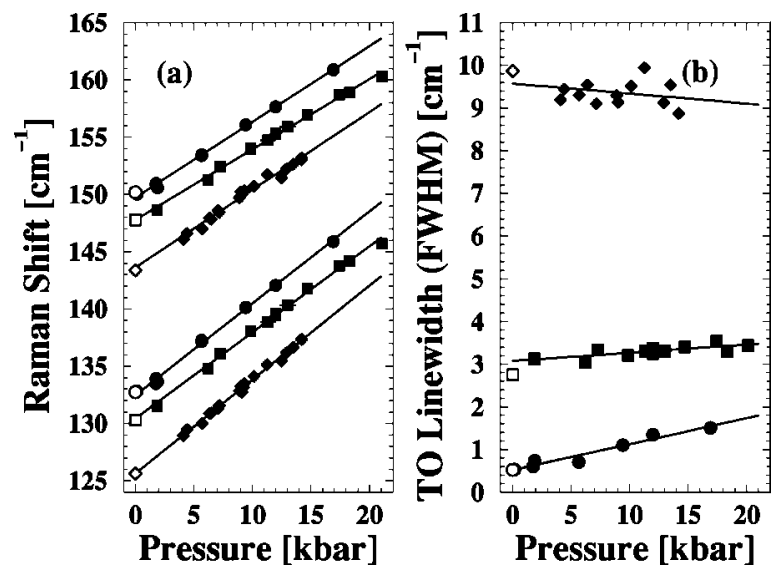

FIG. 7. (a) Pressure dependence of the phonon frequencies and (b) $\mathrm{TO}$ phonon linewidth of ${ }^{63} \mathrm{CuI}$ at $10 \mathrm{~K}$ (circles), $120 \mathrm{~K}$ (squares), and $240 \mathrm{~K}$ (diamonds). Solid symbols display the experimental points whereas the open symbols represent data extracted from Figs. 4 and 5 at 1 bar. The solid lines represent linear fits to the pressure data. For the TO linewidth at $240 \mathrm{~K}$ the points at 1 bar obtained outside the diamond-anvil cell were included in the fit to make up for the lack of data at low pressure.

3 for ambient pressure, and the TO line shape is slightly asymmetric showing a larger spectral weight at low frequencies.

Figure 7(a) shows the measured increase in the TO and LO Raman frequencies vs pressure, as obtained from the spectra of Fig. 6. From the frequency shifts in Fig. 7(a) we derive the Grüneisen parameters at $10 \mathrm{~K} \gamma_{\mathrm{TO}}(\Gamma)=2.5$ and $\gamma_{\mathrm{LO}}(\Gamma)=1.8$ using the expression

$$
\gamma=-\frac{d \ln \omega}{d \ln V}=B \frac{d \ln \omega}{d p},
$$

where $p$ is the pressure and $B$ the bulk modulus (equal to $\kappa_{T}^{-1}$, where $\kappa_{T}$ is the isothermal compressibility). Note that comparison between different sets of Grüneisen parameters is only possible if both sets have been derived using the same value of $B$. We have taken for the bulk modulus $B$ $=40.3 \mathrm{GPa}$ from Ref. 28, although more recent $\mathrm{x}$-ray data yield a value $B=31(2) \mathrm{GPa}^{29}$ In this way, our values of $\gamma$ compare well with those reported by Hochheimer et al. ${ }^{28}$ at $40 \mathrm{~K}, 100 \mathrm{~K}$, and room temperature. ${ }^{30}$ The difference $\gamma_{L O}$ $-\gamma_{T O}(\approx-0.7$ measured for $\mathrm{CuI})$ is related to the dependence of the transverse effective charge $e_{T}^{*}$ and the ionicity on volume (see Ref. 9 and references therein). Using Eq. (3) of Ref. 9 we obtain for $\mathrm{CuI} e_{T}^{*}=1.06$, where $\epsilon_{\infty}=4.84$ was taken from Ref. 31. A Grüneisen parameter $\gamma^{*}$ can be defined to represent the volume dependence of $e_{T}^{*}$, as done in Eq. (4) of Ref. 9. We obtain for $\mathrm{CuI} \gamma^{*}=-1.5$. A negative sign is found for $\gamma^{*}$ in most zinc-blende-type materials (the only known exception ${ }^{32}$ being $\mathrm{SiC}$ ). It corresponds to a decrease in effective charge with increasing pressure.

The analysis of the dependence of phonon linewidths on pressure is more complicated since at different temperatures several distinct decay channels become active. Figure 7(b) displays the pressure dependence of the TO linewidth at 10
$\mathrm{K}$ (solid circles), $120 \mathrm{~K}$ (solid squares), and $240 \mathrm{~K}$ (solid diamonds), together with the ambient pressure data (open symbols) obtained from Fig. 4(a). The solid lines represent linear fits to the frequencies vs pressure, where for $240 \mathrm{~K}$ the 1-bar point from Fig. 4(a) was included to compensate for the lack of data obtained with the diamond-anvil cell at pressures lower than 4 kbar. We notice in Fig. 7(b) a change in the sign of the slopes with increasing temperature that will be discussed in detail in Sec. V with the aid of the theoretical model developed in the next section.

\section{THEORY}

In order to interpret the pressure and temperature dependence of the Raman phonons in $\mathrm{CuI}$ we need to know the appropriate two- and three-phonon densities of states and the dependence of the appropriate van Hove singularities with both pressure and temperature, since they are responsible for the resonances observed in the spectra and the corresponding phonon linewidths and line shifts.

\section{A. Rigid-ion model}

We have employed a rigid-ion force constants model with 11 parameters ${ }^{10}$ fitted to inelastic neutron-scattering data ${ }^{11}$ to calculate the one- $\left[\rho^{(1)}(\omega)\right]$ and two-phonon $\left[\rho^{(2)}(\omega)\right]$ DOS.

The inelastic neutron-scattering data reported for $\mathrm{CuI}$ were obtained at room temperature; we have assumed that the temperature dependence of the different features of the DOS is similar to that reported in Ref. 33 for germanium, rescaled to reproduce the observed temperature dependence of the TO phonons in CuI. The DOS must be scaled by the corresponding Bose-Einstein function when used for modeling the contribution to the self-energy or the linewidth at different temperatures. For the sake of simplicity, a Klemenslike ansatz was used for the different DOS.

In order to represent the anomalous temperature dependence observed for the TO linewidth two different decay channels have been assumed in Sec. III to describe its $T^{2}$ high-temperature behavior, i.e., a two-phonon channel with phonons of different frequencies and a decay into three phonons. Since no information ${ }^{34}$ is available about the threephonon DOS, $\rho^{(3)}(\omega)$, and the one-phonon DOS, $\rho^{(1)}(\omega)$, shows a sharp resonance at $\approx \omega_{T O} / 3$, corresponding approximately to $\mathrm{TA}_{X}$, we have used only a decay channel into three phonons of equal frequency, which implies $\rho^{(3)}(\omega)$ $\propto \rho^{(1)}(\omega / 3)$, and disregarded decays into other three-phonon channels. This simplification, though very rough, yields good qualitative agreement with the observed linewidths vs pressure as will be shown in the discussion.

Another possible decay channel used by Fukumoto et al. ${ }^{23}$ involves a two-phonon difference mode, which simulates a term quadratic in temperature but only at intermediate temperatures. This channel might account for the observed temperature dependence of the TO linewidth at ambient pressure, but cannot reproduce the almost pressure independent linewidth observed at $240 \mathrm{~K}$ and its contribution to the temperature dependence of the LO linewidth is also negated by 
the very low two-phonon difference DOS in the LO region. This issue will be analyzed in more length in Sec. V.

\section{B. Ab initio calculations}

In order to interpret the observed pressure dependence of the phonon line shapes we need the Grüneisen parameters of the acoustic phonons contributing to the decay channels. Little information is available on this subject: The only experimental data for $\mathrm{CuI}$ were obtained by extrapolation of results for the high-pressure tetragonal phase. ${ }^{35}$ We have therefore undertaken a first-principles calculation of the phonon frequencies and their dependence on the lattice constant for the three cuprous halides $(\mathrm{CuCl}, \mathrm{CuBr}$, and $\mathrm{CuI})$. Calculations using the full-potential linear-muffin-tin-orbital (FPLMTO) method ${ }^{36}$ have been reported for the TO Raman phonons, and experimental data for the Grüneisen parameters have been collected in Ref. 35 .

The $a b$ initio calculations were performed with electronic band structures generated within the framework of densityfunctional theory (DFT) and its local-density approximation (LDA), for which we used the Ceperley-Alder form of the exchange-correlation energy density of the homogeneous electron gas as parametrized by Perdew and Zunger. ${ }^{37}$ The present results have been obtained with the ABINIT code, ${ }^{38}$ that is based on a plane-wave representation of the wave functions describing the electron-ion interaction by normconserving pseudopotentials. ${ }^{39,40}$ This representation is able to account for the strong hybridization of the copper $3 d$ and halogen $p$ orbitals. ${ }^{41}$ Phonon frequencies were calculated within linear-response theory ${ }^{42}$ (see Refs. 43 and 44 for technical details concerning Born effective charges and the computation of the dynamical matrix).

A kinetic energy cutoff of 70 hartree was used for the plane-wave expansion and a $4 \times 4 \times 4$ Monkhorst-Pack grid $^{45}$ (ten special points) was employed for integration in the Brillouin zone. With these parameters we obtain a convergence in the total energy better than 1 mhartree. The phonon frequencies are converged to better than $1 \mathrm{~cm}^{-1}$.

Although the LDA yields values of the band gap much lower than the experimental ones (for the cuprous halides the gaps are only $20 \%$ of the experimental values), the calculated phonon frequencies are usually not very sensitive to this so-called "gap effect." In Table II we compare the phonon frequencies calculated at high-symmetry points of the Brillouin zone with those reported by inelastic neutron scattering. ${ }^{11,46,47}$ The experimental values of the lattice constants were used for the calculation of the phonon frequencies and mode Grüneisen parameters; theoretical values of the lattice constants at zero pressure were $\approx 2 \%$ smaller. Calculated and measured phonon frequencies agree within $10 \%$ except for those corresponding to transverse-acoustic phonons ${ }^{48}$ which are more difficult to converge. Our results for the TO phonon frequencies compare well with those reported by FP-LMTO in Ref. 36, although our Grüneisen parameters are $30 \%$ larger. For $\mathrm{CuI}$ the calculated frequencies yield a sign reversal of the TO-LO splitting at the $X$ point, compared to the neutron data. ${ }^{11,24}$ The reduction of the TO-LO splitting at the $\Gamma$ point when going from $\mathrm{CuCl}$ to $\mathrm{CuI}$
TABLE II. Phonon frequencies calculated $a b$ initio for the cuprous halides at high-symmetry points of the Brillouin zone. The experimental lattice constants were used in the calculations. Values in parentheses represent experimental data obtained by inelastic neutron scattering. FP-LMTO data from Ref. 36 are shown in curly brackets. The frequencies are given in $\mathrm{cm}^{-1}$.

\begin{tabular}{cccccc}
\hline \hline & Point & TA & LA & TO & LO \\
\hline $\mathrm{CuCl}$ & $\Gamma$ & 0 & 0 & $148(166)\{156\}$ & $187(207)$ \\
& $X$ & $52(39)$ & $117(124)$ & $211(218)$ & $235(234)$ \\
& $L$ & $42(34)$ & $110(114)$ & $180(192)$ & $244(245)$ \\
$\mathrm{CuBr}$ & $\Gamma$ & 0 & 0 & $134(135)\{136\}$ & $158(169)$ \\
& $X$ & $51(39)$ & $124(126)$ & $158(161)$ & $157(154)$ \\
& $L$ & $40(32)$ & $113(106)$ & $145(150)$ & $167(172)$ \\
$\mathrm{CuI}$ & $\Gamma$ & 0 & 0 & $116(126)\{138\}$ & $132(160)$ \\
& $X$ & $57(46)$ & $115(121)$ & $121(143)$ & $118(156)$ \\
& $L$ & $42(36)$ & $106(100)$ & $118(133)$ & $124(155)$ \\
\hline \hline
\end{tabular}

${ }^{a}$ Experimental data from Ref. 46 measured at $4.2 \mathrm{~K}$.

${ }^{\mathrm{b}}$ Experimental data from Ref. 47 measured at $77 \mathrm{~K}$.

${ }^{\mathrm{c}}$ Experimental data from Ref. 11 measured at room temperature.

is reproduced as well, although one should take into account the difference in temperature between experimental and theoretical data (the former are affected by anharmonic corrections while the latter are not). However, this splitting is much smaller in the frequencies calculated for CuI. The calculated splitting can be increased if the band gap is corrected and the screening reduced, but this would not affect the TO-LO splittings at other points of the Brillouin zone, where differences with the experimental data can be due to anharmonic effects. Another important feature observed in Table II is that the frequencies of the TO and $\mathrm{LO}$ phonons decrease with the mass of the halogen, whereas those of the acoustic phonons remain nearly constant for all cuprous halides. This explains why the observed ${ }^{5,8}$ Fermi resonances for $\mathrm{CuCl}$ and $\mathrm{CuBr}$ appear at the TO and LO frequencies, respectively, since the relevant van Hove singularity of the two-phonon DOS responsible for those resonances corresponds to a TA $+\mathrm{LA}$ phonon combination.

Table III displays the Grüneisen parameters $\gamma$ calculated for the cuprous halides at different high-symmetry points of the Brillouin zone, together with experimental data (in parentheses) collected in Ref. 35 and more recent data from Refs. 6 and 9. The experimental parameters obtained from our Raman data vs pressure for the $\Gamma$ point at $10 \mathrm{~K}$ are also shown in brackets. The calculated $\gamma$ 's were obtained from linear fits to the dependence of $\ln (\omega)$ on $\ln (a)$, with $a$ the lattice constant, for three different lattice constants around the experimental value. Although the calculated Grüneisen parameters are $\approx 30 \%$ larger than those extracted from our pressure measurements, we can use them to interpret qualitatively differences between the three cuprous halides. Note that the negative sign of $\gamma_{L O}-\gamma_{T O}$ observed for the Raman phonons is also reproduced by the calculation. For the effective charge of $\mathrm{CuI}$ we obtain $e_{T}^{*}=1.24$, which also agrees reasonably with the value extracted from our measurements $(\approx 1.06$ for $10 \mathrm{~K})$. The slightly higher calculated value of $e_{T}^{*}$ may be related to an overestimate of $\epsilon_{\infty}$, attributed to the 
TABLE III. Mode Grüneisen parameters for the cuprous halides at high-symmetry points of the Brillouin zone calculated using the linear response method within density functional perturbation theory. Values in brackets denote our experimental results for the zone center modes converted from pressure derivatives to volume derivatives using a bulk modulus $B=40.3 \mathrm{GPa}$ (Refs. 28 and 30). Experimental data in parentheses were reported in Refs. 6, 9, and 35, and FP-LMTO data from Ref. 36 are shown in curly brackets.

\begin{tabular}{lccccc}
\hline \hline & & TA & LA & TO & LO \\
\hline $\mathrm{CuCl}$ & $\Gamma$ & & & $4.3(2.4)(1.9)^{\mathrm{a}}\{3.2\}$ & $2.5(1.0)(1.5)^{\mathrm{a}}$ \\
& $X$ & $-0.7(-1.4)^{\mathrm{b}}$ & 2.3 & 2.7 & 1.9 \\
& $L$ & $-1.5(-1.4)^{\mathrm{b}}$ & 1.8 & 3.3 & 2.0 \\
$\mathrm{CuBr}$ & $\Gamma$ & & & $3.4(2.4)(1.8)^{\mathrm{c}}\{2.8\}$ & $2.3(1.9)(1.2)^{\mathrm{c}}$ \\
& $X$ & $-1.5(-0.4)^{\mathrm{b}}$ & 2.2 & 3.0 & 1.9 \\
& $L$ & $-1.9(-0.4)^{\mathrm{b}}$ & 1.4 & 3.3 & 2.2 \\
$\mathrm{CuI}$ & $\Gamma$ & & & $3.2[2.5]\{2.5\}$ & $2.3[1.8]$ \\
& $X$ & $-0.3(2.5)^{\mathrm{d}}$ & 2.2 & 3.5 & 2.9 \\
& $L$ & $-0.9(2.5)^{\mathrm{d}}$ & 1.5 & 3.5 & \\
\hline \hline
\end{tabular}

${ }^{\mathrm{a}}$ Raman data reported in Ref. 6.

${ }^{b}$ From Raman data for $\mathrm{CuCl}_{0.80} \mathrm{Br}_{0.20}$ and $\mathrm{CuCl}_{0.20} \mathrm{Br}_{0.80}$ alloys (Ref. 52).

${ }^{\mathrm{c}}$ Raman data reported in Ref. 9.

${ }^{\mathrm{d}}$ Extrapolated from Raman data of the high-pressure tetragonal phase (Ref. 15).

band-gap problem of the LDA. However, the calculation of $\gamma^{*}$ yields too large a value, as expected from the small calculated TO-LO splitting.

The calculated $\gamma_{T A}$ shows a negative sign for all copper halides, however, the value for $\mathrm{CuI}$ is very small. According to Ref. 35 a decrease of the magnitude of the Grüneisen parameters and even a change of sign is expected for the transverse acoustic phonons when going through the series $\mathrm{CuCl}-\mathrm{CuBr}-\mathrm{CuI}$. This result is further supported by the change of sign of the slope in the temperature dependence of the lattice parameter for $\mathrm{CuCl}$ and $\mathrm{CuBr}$, which does not appear $^{49}$ in $\mathrm{CuI}$ and indicates a small magnitude or a positive sign of $\gamma_{T A}$ for this compound. Our calculations shows also a decrease of $\gamma_{T A}$ from $\mathrm{CuCl}$ and $\mathrm{CuBr}$ to $\mathrm{CuI}$. This trend is different from that observed in II-VI compounds such as the series $\mathrm{ZnS}-\mathrm{ZnSe}-\mathrm{ZnTe}$, where ${ }^{35} \gamma_{T A}$ (always negative) increases in magnitude for the $X$ point and decreases slightly but remains negative for the $L$ point. The calculated data of Table III fail to explain the value of 2.5 reported in Ref. 35 for $\mathrm{CuI}$ for the transverse acoustic Grüneisen parameters at the $X, K$, and $L$ points. However, this value was obtained by extrapolation of Raman measurements for the high-pressure tetragonal phase ${ }^{15}$ and from disordered alloys; ${ }^{22}$ these procedures are obviously questionable. Precise measurements of the second-order Raman spectra under pressure would be required to obtain experimental data which can be reasonably compared with our calculated values. We have therefore used our calculated Grüneisen parameters to model the pressure dependence of the two- and three-phonon DOS obtained with the rigid-ion model, and thus investigate and explain the pressure dependence of the Raman spectra measured at different temperatures.

\section{DISCUSSION}

The low-temperature spectra of Fig. 1 can be basically understood within the virtual crystal approximation, as ex- plained in Sec. II. However, we have seen that the introduction of the anharmonic terms of Eqs. (1) and (2) improves slightly the fits in Fig. 2. This is in agreement with the lowtemperature Raman data reported for $\mathrm{CuBr}^{8}{ }^{8}$ Moreover, the fits yield values of the zero-temperature renormalization in agreement with those obtained by linear extrapolation of the phonon frequencies vs temperature to $0 \mathrm{~K}$.

In order to analyze in detail the different aspects of the temperature dependence of the measured spectra, we have plotted in Fig. 8(a) the Raman spectrum for ${ }^{65} \mathrm{CuI}$ at $170 \mathrm{~K}$, as indicative of the high-temperature behavior observed in Fig. 3. The lowest frequency structure in this spectrum is a broad band located at $\approx 95 \mathrm{~cm}^{-1}$. This structure has been asigned in the past ${ }^{22,23}$ to a $2 \mathrm{TA}$ phonon combination enhanced by disorder effects within an off-center model for the copper ions. This off-center model has been discarded rather conclusively in recent work with isotopically substituted samples. ${ }^{5,6,8}$ The fact that this feature appears at high temperatures (see Fig. 3), and the low frequencies reported by inelastic neutron scattering for the transverse phonons at the zone boundaries have led us to assign this feature to two phonon difference modes. Figure 8 (b) displays as a thick line the two-phonon difference DOS $\left[\rho_{-}^{(2)}(\omega)\right]$, which exhibits a pronounced van Hove singularity centered at $\approx 94 \mathrm{~cm}^{-1}$, arising mainly from (TO-TA) $)_{L}$ phonon differences. This singularity supports our interpretation of the spectral weight in this region at high temperatures.

The slightly asymmetric line shape exhibited by the TO peak at high temperatures (see arrows in Fig. 3), and the fact that the TO linewidth observed at room temperature is larger for ${ }^{63} \mathrm{CuI}$ than for ${ }^{65} \mathrm{CuI}$, can be also attributed to a resonance with the two-phonon difference DOS. In Fig. 8(b) a broad structure appears in the range between $\approx 104$ and $125 \mathrm{~cm}^{-1}$, with a kink at its high-frequency side. This band has three van Hove singularities, denoted by A, B, and C in the figure, at 103,113 , and $124 \mathrm{~cm}^{-1}$, which are related to 

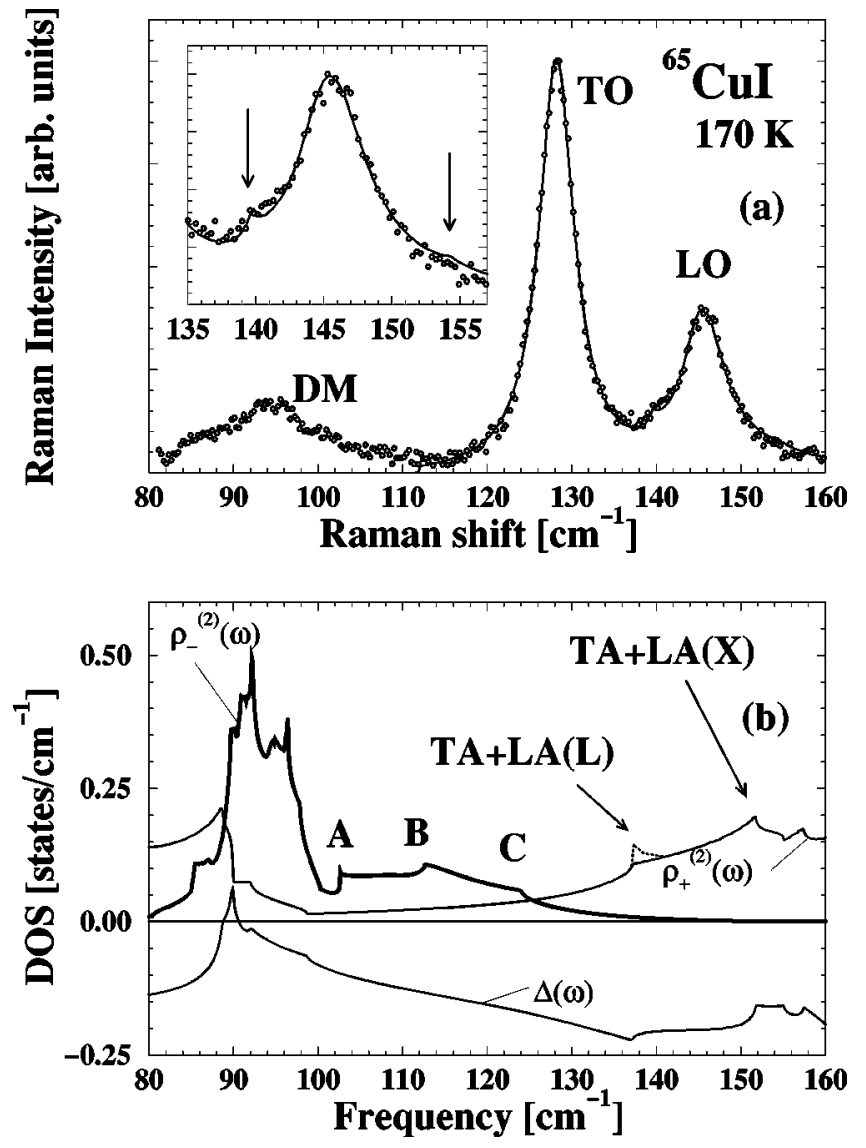

FIG. 8. (a) Measured Raman spectra of ${ }^{65} \mathrm{CuI}$ at $170 \mathrm{~K}$ and (b) graph with the calculated two-phonon difference $\left[\rho_{-}^{(2)}(\omega)\right.$, thick line $]$ and sum $\left[\rho_{+}^{(2)}(\omega)\right]$ DOS, together with the Kramers-Kronig transform of the latter $[\Delta(\omega)]$, using a rigid-ion model. In (a) the circles represent the experimental data and the solid line displays a fit assuming a Fermi resonance model as used in Refs. 5 and 8 for low-temperature Raman spectra of $\mathrm{CuCl}$ and $\mathrm{CuBr}$, respectively. A broad feature is observed at $\approx 95 \mathrm{~cm}^{-1}$ that can be attributed to difference modes, as shown in the lower graph. The inset in the upper graph shows more clearly the resonance at the LO peak and the arrows denote the position of van Hove singularities of $\rho_{+}^{(2)}(\omega)$ at $\mathrm{TA}+\mathrm{LA}_{L}$ and $\mathrm{TA}+\mathrm{LA}_{X}$. The dotted peak in the lower graph at $\approx 139 \mathrm{~cm}^{-1}$ represents an ad hoc modification of $\rho_{+}^{(2)}(\omega)$ required to account for the shoulder observed at the LO peak.

the difference modes $(\text { TO-TA })_{L}, \quad(\text { LO-TA })_{X}$, and $(\mathrm{LO}-\mathrm{TA})_{L}$, respectively. The latter would be responsible for the increase of the TO linewidth of $\approx 2 \mathrm{~cm}^{-1}$ at room temperature observed for ${ }^{63} \mathrm{CuI}$ with respect to ${ }^{65} \mathrm{CuI}$. From the mass dependence of the Raman phonons one would expect the TO peak of ${ }^{65} \mathrm{CuI}$ to shift down in frequency with increasing temperature faster than that of ${ }^{63} \mathrm{CuI}$. However, the eigenvectors of the LO phonons at the zone boundary have usually a larger component of copper mass than the TO phonon at the $\Gamma$ point. Moreover, the absolute shift to lower frequencies should be larger due to the larger frequency of the LO phonons. In summary, with increasing temperature $\rho_{-}^{(2)}(\omega)$ shifts to lower frequencies faster than the Raman phonons, therefore the coupling is weaker for ${ }^{65} \mathrm{CuI}$ and the TO linewidth is smaller than that of ${ }^{63} \mathrm{CuI}$.
In Fig. 8(a) we can also observe a shoulder at the lowfrequency side of the LO peak, already mentioned in Sec. II. This feature can be reproduced by the Fermi resonance model introduced by Krautzman et al. ${ }^{1}$ in a way similar to that employed in Refs. 5 and 8 to explain the anomalous low-temperature line shape of the $\mathrm{TO}$ and $\mathrm{LO}$ peaks of $\mathrm{CuCl}$ and $\mathrm{CuBr}$, respectively. We have already pointed out that the two-acoustic-phonon (sum) DOS, $\rho_{+}^{(2)}(\omega)$, remains almost unchanged from $\mathrm{CuCl}$ to $\mathrm{CuBr}$, as can be inferred from the zone-edge phonon frequencies displayed in Table II. The $M_{1}$-type singularity seen in Fig. 8(b) at $137 \mathrm{~cm}^{-1}$ is due to the sum of TA and LA phonons at the $L$ point of the Brillouin zone, and can be used, together with its Hilbert transform (dashed line), to fit the line shape of the LO structure. In the spirit of Refs. 5 and 8, we model the linewidth with the equation

$$
\Gamma(\omega)=\left|V_{3}\right|^{2} \rho_{0}^{(2)}(\omega)\left[1+2 n_{B E}(\omega / 2 T)\right],
$$

where $\left|V_{3}\right|$ is a third-order anharmonic coupling parameter and we have included a Bose-Einstein factor to account for the finite temperature. $\rho_{0}^{(2)}(\omega)$ is the two-phonon (sum) DOS for $0 \mathrm{~K}$, obtained from that calculated in Sec. IV B for room temperature by scaling with the temperature dependence of the different phonons in germanium. The fit procedure has been described elsewere ${ }^{5,8,9,50}$ and yields for $\left|V_{3}\right|^{2}$ a value of $\approx 6 \mathrm{~cm}^{-2}$. The $(\mathrm{TA}+\mathrm{LA})_{L}$ singularity has been enhanced ad hoc [dotted line in Fig. 8(b)] in order to better reproduce the shape of the shoulder. Similar enhancement also had to be invoked to explain the dependence of the linewidth of diamond on isotopic composition ${ }^{51}$ in spite of the rather good knowledge of the dispersion relations of this material as compared with those of the cuprous halides. The solid line in Fig. 8(a) represents a fit with Eq. (7) and with Eq. (2) of Ref. 8 for the spectrum of ${ }^{65} \mathrm{CuI}$ at $170 \mathrm{~K}$. The inset of this figure shows good agreement between the fit and the experimental data; the arrows indicate the frequency of the singularities of $\rho_{+}^{(2)}(\omega)$ labeled in Fig. 8(b).

The temperature dependence of the LO phonon linewidth can be summarized as follows: The LO phonons display a normal anharmonic behavior that can be mainly attributed to decay into two acoustic phonons as shown by the fits in Fig. 4. Difference modes are ruled out, contrary to the interpretation of Fukumoto et al., on the basis of the low density of two-phonon difference states displayed in Fig. 8(b) for that frequency region. We ascribe the discrepancy between the fit and the experimental data in Fig. 4(b) for ${ }^{65} \mathrm{CuI}$ to an artifact of the deconvolution procedure related to the lower resolution employed in those measurements. This discrepancy might also arise from an additional decay channel into three acoustic phonons, as used for the TO peak, but there is not enough evidence to support such hypothesis.

Figure 9 shows several plots of $\rho_{+}^{(2)}(\omega)$ and $\rho^{(3)}(\omega)$ for different pressure and temperature conditions (i.e., including the appropriate Bose-Einstein factors). The thick solid curve represents a three-phonon density of states obtained under the assumption of Klemens-type processes in which the 3 phonons involved have the same frequency. This curve thus corresponds to the one-phonon density of states with the fre- 

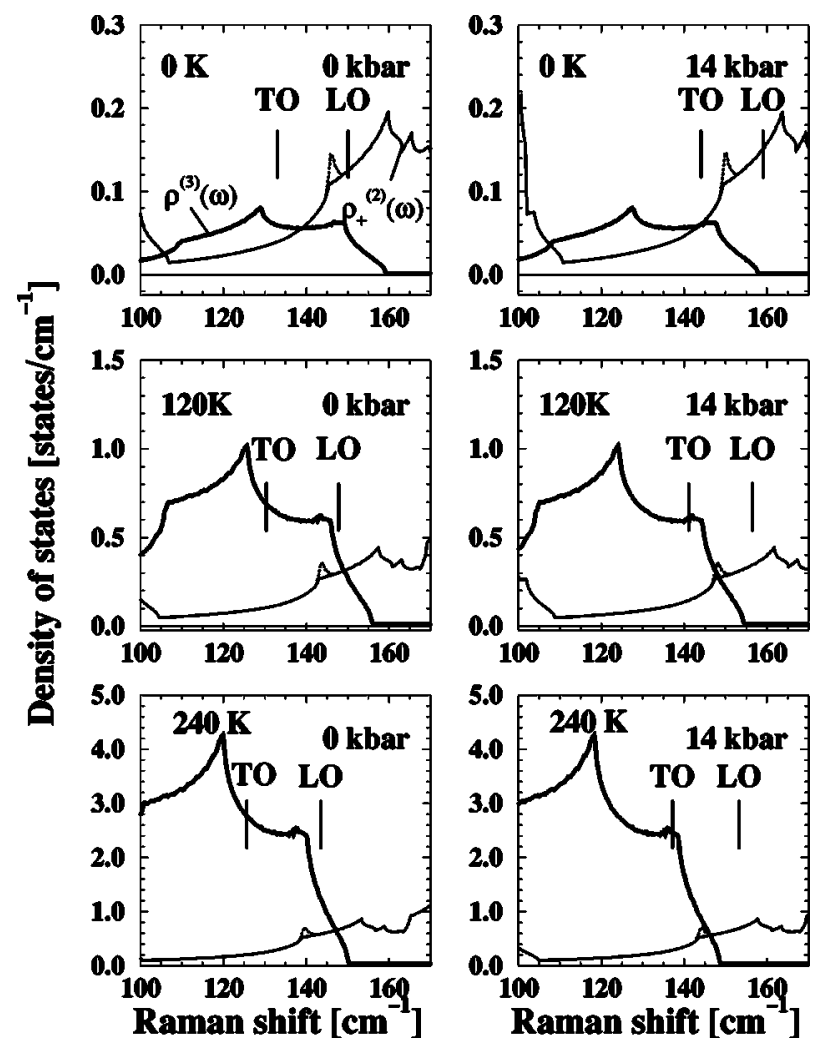

FIG. 9. Two- $\left[\rho_{+}^{(2)}(\omega)\right]$ and three-phonon $\left[\rho^{(3)}(\omega)\right.$, thick line $]$ DOS obtained with the 11 parameter rigid-ion model of Ref. 10. $\rho^{(3)}(\omega)$ was generated by scaling the one-phonon DOS assuming a decay into three phonons of equal frequency. Both DOS, obtained from neutron data at room temperature, have been shifted horizontally, and scaled vertically by the corresponding Bose-Einstein function, in order to represent the different temperature and pressure conditions. In addition, $\rho^{(3)}(\omega)$ has been divided by a factor of 2 in order to better display both DOS in the same graph.

quency scale expanded by a factor of three. We observe in $\rho^{(3)}(\omega)$ a broad structure centered at $\approx 130 \mathrm{~cm}^{-1}$ related to three TA phonons at the $X$ and $W$ points. The resonance of the TO phonon with this structure should be responsible for the anomalous increase in linewidth with temperature. The fact that higher-order anharmonic terms are not expected to contribute significantly at low temperatures explains the very narrow width $\left(\approx 0.5 \mathrm{~cm}^{-1}\right)$ observed at $2 \mathrm{~K}: \rho_{+}^{(2)}(\omega)$ is very small in the region. These DOS have been rigidly shifted and scaled by the corresponding Bose-Einstein functions to take into account the effect of temperature, as described in the previous section. The Grüneisen parameters calculated $a b$ initio $\left(\gamma_{\mathrm{TA}+\mathrm{LA}} \simeq 0.85\right.$ and $\left.\gamma_{3 T A} \simeq-0.9\right)$ have been employed to rigidly shift $\rho_{+}^{(2)}(\omega)$ and $\rho^{(3)}(\omega)$ with pressure. This approximation is reasonable in the vicinity of the singularities (see vertical lines in Fig. 9).

Using the Grüneisen parameters of Table III and the modeled DOS displayed in Fig. 9, we can explain qualitatively the trends observed in Figs. 6 and 7 for the pressure dependence of the Raman phonons of ${ }^{63} \mathrm{CuI}$. Because of the small Grüneisen parameter of the $(\mathrm{TA}+\mathrm{LA})_{L}$ phonon combination, the TO phonon, at $133 \mathrm{~cm}^{-1}$ for $p=1 \mathrm{bar}$, will shift towards the TA+LA singularity thus giving rise to an in-
TABLE IV. Pressure derivatives (in $\mathrm{cm}^{-1} / \mathrm{GPa}$ ) of the TO phonon linewidth of ${ }^{63} \mathrm{CuI}$ at different temperatures. Experimental data from Fig. 7 are compared with those obtained from the two- and three-phonon DOS of Fig. 9 and Eq. (8). The values of the anharmonic coefficients $\left|V_{3}\right|^{2}=8.8 \mathrm{~cm}^{-2}$ and $\left|V_{4}\right|^{2}=1.46 \mathrm{~cm}^{-2}$ were extracted from the fit to the temperature dependence of the corresponding linewidth, shown in Fig. 4.

\begin{tabular}{lccc}
\hline \hline & \multicolumn{3}{c}{$T[\mathrm{~K}]$} \\
& 10 & 120 & 240 \\
\hline Expt. & $0.61(6)$ & $0.19(5)$ & $-0.2(2)$ \\
Theor. & 0.12 & 0.10 & -0.16 \\
\hline \hline
\end{tabular}

crease in the linewidth in qualitative agreement with the data of Fig. 4 [see also similar results obtained for the Raman phonons of silicon under pressure ${ }^{50}$ and the TO mode in $\mathrm{CuBr}$ (Ref. 9)]. The shift of $\mathrm{TO}(\Gamma)$ away from the threephonon singularity will contribute a nearly negligible decrease in width with increasing pressure at $10 \mathrm{~K}$. At $240 \mathrm{~K}$, however, the three-phonon contribution becomes dominant [actually $\rho^{(3)}(\omega)$ has been divided by a factor of 2 in the graphs of Fig. 9 to better display both densities of states in the same plots] and it is much larger than the two-phonon term, a fact which was already invoked to explain the linewidths in Fig. 4. When applying pressure, however, the TO frequency shifts up with respect to either singularity. This shift results, according to Fig. 9, in a decrease of the threephonon contribution and an increase of the two-phonon term to the TO width. The compensation must be nearly complete in order to explain the nearly pressure independent width observed at $240 \mathrm{~K}$ in Figs. 6 and 7.

In order to make quantitative predictions of the pressure dependence of the linewidth displayed in Fig. 7, we have used the information obtained from the temperature dependence measurements, in the sense that the linewidth can be expressed as a sum of a third-order term and a fourth-order contribution. In the spirit of Ref. 5, these contributions can be represented by ad hoc anharmonic constants multiplying the corresponding density of states responsible for the decay process:

$$
\Gamma(\omega)=\left|V_{3}\right|^{2} \rho_{+}^{(2)}(\omega)+\left|V_{4}\right|^{2} \rho^{(3)}(\omega) .
$$

The values of the coefficients $\left|V_{3}\right|^{2}$ and $\left|V_{4}\right|^{2}$ are uniquely determined when we require that Eq. (8) yields the parameters $\Gamma_{1}$ and $\Gamma_{2}$ of the fit for the TO phonon linewidth with Eq. (4). In Table IV we display the pressure dependence of the TO linewidth extracted from the analysis of Fig. 9. Notice that although the estimated magnitude is smaller, the DOS analysis reproduces the sign reversal of the slopes observed in Fig. 7. The change with temperature for constant pressure is included automatically through Bose-Einstein factors. The calculation of the TO phonon linewidth at low temperature using Eq. (8) is strongly affected by small deviations in the relative position of $\rho_{+}^{(2)}(\omega)$ with respect to the Raman phonons. Typical deviations of $\pm 5 \mathrm{~cm}^{-1}$ that are expected from the error bars of the inelastic neutronscattering data would explain the discrepancy shown for 10 
$\mathrm{K}$ between measured and calculated values of the pressure dependences of the TO phonon linewidth displayed in Table IV. The discrepancy between calculated and measured Grüneisen parameters can also contribute to this difference in the slopes. The use of the experimental Grüneisen parameters for the TA modes would even worsen this situation. The value of $\left|V_{3}\right|^{2} \simeq 8.8 \mathrm{~cm}^{-2}$ obtained from this analysis is significantly smaller than those reported for $\mathrm{CuCl}\left(70 \mathrm{~cm}^{-2}\right.$, Ref. 5) and $\mathrm{CuBr}\left(45 \mathrm{~cm}^{-2}\right.$, Ref. 8). This reveals clearly the decrease in anharmonicity throughout the series $\mathrm{CuCl}-\mathrm{CuBr}-\mathrm{CuI}$, i.e., with increasing halogen mass.

\section{CONCLUSIONS}

We have performed a comprehensive investigation of the Raman phonons under different temperature and pressure conditions in isotopically substituted $\gamma$-CuI. At low temperature both TO and LO phonons behave with copper mass mainly as predicted by the virtual crystal approximation, slight deviations from this model can be accounted for by the lowest order anharmonic contributions.

The TO phonon linewidth exhibits an anomalous temperature dependence that can be best understood in terms of a decay into two and three acoustic phonons. The LO phonon linewidth is rather normal, i.e., it can be explained by decay into two acoustic phonons. Nevertheless, at high temperatures, a shoulder develops at its low-energy side which can be attributed to a resonance with the two-phonon sum density of states. To model these line shapes and their dependence on pressure and temperature, the one- and two-phonon densities of states were obtained using a rigid-ion model, and first-principles calculations were performed for the phonon frequencies and Grüneisen parameters. The calculated values agree qualitatively with those available in the literature and allow the interpretation and explanation of the TO linewidth and its change with pressure as related to a resonance with a three TA phonon combination. Difference modes have been discovered at 95 and $124 \mathrm{~cm}^{-1}$ which explain the broad structure and an additional broadening of the TO peak observed in the high-temperature spectra of ${ }^{63} \mathrm{CuI}$ as compared to that of ${ }^{65} \mathrm{CuI}$. The Grüneisen parameters calculated for the transverse-acoustic phonons, although negative, reflect the smaller magnitude reported in the literature for $\mathrm{CuI}$ as compared to those of $\mathrm{CuCl}$ and $\mathrm{CuBr}$. This trend contrasts with the behavior observed for the related II-VI series, $\mathrm{ZnS}-$ $\mathrm{ZnSe}-\mathrm{ZnTe}$, where the magnitude of the negative Grüneisen parameters for the transverse-acoustic phonons tends to increase from $\mathrm{ZnS}$ to $\mathrm{ZnTe}$.

\section{ACKNOWLEDGMENTS}

The authors have benefited from valuable discussions with A. Cantarero, F. Widulle, and C. Ulrich. J.S. acknowledges financial support from the Max-Planck-Gesellschaft and the Ministerio de Educación, Cultura y Deportes (Spain) through the Plan Nacional de Formación del Profesorado Universitario. A.R. was partially supported by DGES, University of the Basque Country, and EC-RTN programs Nanophase (HPRN-CT-2000-00167) and Comelcan (HPRNCT-2000-00128). Computer time has been granted by the Center de Computació i Comunicacions de Catalunya $\left(\mathrm{C}^{4}\right)$. Financial support from the Fonds der Chemischen Industrie is also gratefully acknowledged. We thank S. R. Stambach for assistance with the pressure measurements and I. Loa and F. J. Manjón for a critical reading of the manuscript.
${ }^{1}$ M. Krauzman, R.M. Pick, H. Poulet, G. Hamel, and B. Prevot, Phys. Rev. Lett. 33, 528 (1974).

${ }^{2}$ Z. Vardeny and O. Brafman, Phys. Rev. B 19, 3276 (1979).

${ }^{3}$ C.H. Park and D.J. Chadi, Phys. Rev. Lett. 76, 2314 (1996).

${ }^{4}$ S.R. Bickham, J.D. Kress, L.A. Collins, and R. Stumpf, Phys. Rev. Lett. 83, 568 (1999).

${ }^{5}$ A. Göbel, T. Ruf, C.-T. Lin, M. Cardona, J.-C. Merle, and M. Joucla, Phys. Rev. B 56, 210 (1997).

${ }^{6}$ C. Ulrich, A. Göbel, K. Syassen, and M. Cardona, Phys. Rev. Lett. 82, 351 (1999).

${ }^{7}$ G. Kanellis, W. Kress, and H. Bilz, Phys. Rev. Lett. 56, 938 (1986); Phys. Rev. B 33, 8724 (1986); 33, 8733 (1986).

${ }^{8}$ J. Serrano, T. Ruf, F. Widulle, C.T. Lin, and M. Cardona, Phys. Rev. B 64, 045201 (2001).

${ }^{9}$ F.J. Manjón, J. Serrano, I. Loa, K. Syassen, C.T. Lin, and M. Cardona, Phys. Rev. B 64, 064301 (2001).

${ }^{10}$ K. Kunc, M. Balkanski, and M.A. Nusimovici, Phys. Status Solidi B 72, 229 (1975).

${ }^{11}$ B. Hennion, F. Moussa, B. Brevot, C. Carabatos, and C. Schwab, Phys. Rev. Lett. 28, 964 (1972).

${ }^{12}$ C.T. Lin, E. Schönherr, A. Schmeding, T. Ruf, A. Göbel, and M. Cardona, J. Cryst. Growth 167, 612 (1996).
${ }^{13}$ J. Serrano, Ch. Schweitzer, C.T. Lin, K. Reimann, M. Cardona, and D. Fröhlich, Phys. Rev. B 65, 125110 (2002).

${ }^{14}$ C. T. Lin et al. (unpublished).

${ }^{15}$ O. Brafman, M. Cardona, and Z. Vardeny, Phys. Rev. B 15, 1081 (1977).

${ }^{16}$ C. Ulrich, Ph.D. dissertation, University of Stuttgart, 1997 (unpublished).

${ }^{17}$ L. Viña, S. Logothetidis, and M. Cardona, Phys. Rev. B 30, 1979 (1984).

${ }^{18}$ B.A. Weinstein, Philos. Mag. B 50, 709 (1984).

${ }^{19}$ J. Menéndez and M. Cardona, Phys. Rev. B 29, 2051 (1984).

${ }^{20}$ F. Widulle, T. Ruf, M. Konuma, I. Silier, M. Cardona, W. Kriegseis, and V.I. Ozhogin, Solid State Commun. 118, 1 (2002).

${ }^{21}$ M. Cardona, in Light Scattering in Solids, edited by M. Cardona and G. Güntherodt (Springer-Verlag, Berlin, 1982), Vol. 2, p. 49.

${ }^{22}$ Z. Vardeny and O. Brafman, Phys. Rev. B 19, 3290 (1979).

${ }^{23}$ T. Fukumoto, K. Tabuchi, S. Nakashima, and A. Mituishi, Opt. Commun. 10, 78 (1974).

${ }^{24}$ H. Bilz and W. Kress, in Phonon Dispersion Relations in Insulators, edited by M. Cardona, P. Fulde, and H.-J. Queisser (Springer-Verlag, Berlin, 1979), p. 116.

${ }^{25}$ These TA frequencies were scaled by the ratio of Raman (at $2 \mathrm{~K}$ ) 
to neutron-scattering (at $300 \mathrm{~K}$ ) frequencies (Ref. 11).

${ }^{26}$ P.G. Klemens, Phys. Rev. 148, 845 (1966).

${ }^{27}$ H. Herchen and M.A. Cappelli, Phys. Rev. B 47, 14193 (1993).

${ }^{28}$ H.D. Hochheimer, M.L. Shand, J.E. Potts, R.C. Hanson, and C.T. Walker, Phys. Rev. B 14, 4630 (1976).

${ }^{29}$ M. Hofmann, S. Hull, and D.A. Keen, Phys. Rev. B 51, 12022 (1995).

${ }^{30}$ In Ref. 28 temperature-dependent isothermal compresibilities are given for 40, 100, and $295 \mathrm{~K}$. Using these data for calculating the bulk modulus, we obtain from the slopes of Fig. 7 the Grüneisen parameters $\gamma_{\mathrm{TO}}=2.5,2.3$, and 2.4, and $\gamma_{\mathrm{LO}}=1.8,1.7$, and 1.8 for 10,120 , and $240 \mathrm{~K}$, respectively. These values agree rather well with those reported in Ref. 28.

${ }^{31}$ J.E. Potts, R.C. Hanson, and C.T. Walker, Solid State Commun. 13, 389 (1973).

${ }^{32}$ D. Olego, M. Cardona, and P. Vogl, Phys. Rev. B 25, 3878 (1982).

${ }^{33}$ G. Nelin and G. Nilsson, Phys. Rev. B 10, 612 (1974).

${ }^{34}$ Note that the calculation of $\rho^{(3)}(\omega)$ implies summing up frequencies of three phonons $\omega_{1}\left(\mathbf{q}_{1}\right), \omega_{2}\left(\mathbf{q}_{2}\right)$, and $\omega_{3}\left(\mathbf{q}_{3}\right)$ satisfying momentum conservation, i.e., $\mathbf{q}_{1}+\mathbf{q}_{2}+\mathbf{q}_{3}=0$, which is by no means trivial.

${ }^{35}$ B. A. Weinstein and R. Zallen, in Light Scattering in Solids, edited by M. Cardona and G. Güntherodt (Springer-Verlag, Berlin, 1984), Vol. 4, p. 472.

${ }^{36}$ J.W. Kremer and K.H. Weyrich, Phys. Rev. B 40, 9900 (1989).

${ }^{37}$ D.M. Ceperley and B.J. Alder, Phys. Rev. Lett. 45, 566 (1980); J.P. Perdew and A. Zunger, Phys. Rev. B 23, 5048 (1981).

${ }^{38}$ The ABINIT code is a common project of the Universite Catholique de Louvain, Corning Incorporation, and other contributors (URL http://www.abinit.org).

${ }^{39}$ N. Troullier and J.L. Martins, Phys. Rev. B 43, 1993 (1991).

${ }^{40} \mathrm{We}$ used the following core radii in the generation of the pseudo- potentials (in bohrs): $\mathrm{Cu}: 2.05\left(4 s^{1}\right.$ and $\left.3 d^{10}\right)$ and $2.30\left(4 p^{0}\right)$; Cl: $1.65\left(3 s^{2}, 3 p^{5}\right.$, and $\left.3 d^{0}\right)$; $\mathrm{Br}: 1.85\left(4 s^{2}, 4 p^{5}\right.$, and $\left.4 d^{0}\right)$; I: $2.85\left(5 s^{2}, 5 p^{5}\right.$, and $\left.5 d^{0}\right)$.

${ }^{41}$ Similar pseudopotentials have been utilized to investigate phase transitions and the electronic structure in silver halides. See G.S. Nunes, P.B. Allen, and J.L. Martins, Phys. Rev. B 57, 5098 (1998); Solid State Commun. 105, 377 (1998).

${ }^{42}$ S. Baroni, S. de Gironcoli, A. Dal Corso, and P. Giannozzi, Rev. Mod. Phys. 73, 515 (2001).

${ }^{43}$ X. Gonze, Phys. Rev. B 55, 10337 (1997).

${ }^{44}$ X. Gonze and C. Lee, Phys. Rev. B 55, 10355 (1997).

${ }^{45}$ H.J. Monkhorst and J.D. Pack, Phys. Rev. B 8, 5747 (1973).

${ }^{46}$ B. Prevot, B. Hennion, and B. Dorner, J. Phys. C 10, 3999 (1977).

${ }^{47}$ S. Hoshino, Y. Fujii, J. Harada, and J.D. Axe, J. Phys. Soc. Jpn. 41, 965 (1976).

${ }^{48}$ Note that the differences between experimental frequencies and those calculated in Ref. 36 is only 3\%: The LMTO treatment is more adequate for the $3 d$ electrons than that based on planewave pseudopotentials.

${ }^{49}$ Landolt-Börnstein, Numerical Data and Functional Relationships in Science and Technology, Group III, Vol. 17, Pt. b (SpringerVerlag, Berlin, 1982), p. 501.

${ }^{50}$ C. Ulrich, E. Anastassakis, K. Syassen, A. Debernardi, and M. Cardona, Phys. Rev. Lett. 78, 1283 (1996).

${ }^{51}$ K.C. Hass, M.A. Tamor, T.R. Anthony, and W.F. Banholzer, Phys. Rev. B 45, 7171 (1992); J. Spitzer, P. Etchegoin, M. Cardona, T.R. Anthony, and W.F. Banholzer, Solid State Commun. 88, 509 (1993).

${ }^{52}$ B. J. Parsons and C. D. Clark in Light Scattering in Solids, edited by M. Balkanski, R. C. C. Leite, and S. P. S. Porto (Flammarion, Paris, 1976), p. 414. 